\begin{tabular}{|c|c|c|c|c|c|c|c|c|}
\hline 5 Bevölkerung & 1850 & 1880 & 1900 & 1930 & $19+1$ & 1950 & 1960 & $\begin{array}{c}\mathrm{Zu}-/ \text { Abnahme } \\
1850-1960 \\
\%\end{array}$ \\
\hline Arlesheim & 910 & 947 & 1599 & 3228 & 3360 & 3898 & 5219 & 474 \\
\hline Dornach & 840 & 1169 & 1543 & 3067 & 3056 & 3572 & 4260 & 4o7 \\
\hline Reinach & 816 & 951 & 1213 & 2558 & 2813 & 3475 & 6152 & 654 \\
\hline Grellingen & 512 & 1032 & 1012 & 1085 & 1067 & 1160 & 1399 & 173 \\
\hline Muttenz & $1322 *$ & 2057 & 2502 & 4966 & 5929 & 7125 & 11963 & 805 \\
\hline Pratteln & 1371 & 1868 & 2425 & +782 & $51+2$ & 6863 & 9492 & 592 \\
\hline Gempen & 378 & 376 & 355 & 345 & 308 & 301 & 250 & -34 \\
\hline Hochwald & 624 & 588 & 582 & 479 & 448 & 463 & 463 & -26 \\
\hline Seewen & 933 & 823 & 762 & 805 & 840 & 869 & 844 & -10 \\
\hline Büren & 611 & 559 & 546 & 531 & 568 & 548 & 561 & -8 \\
\hline Nuglar-St. Pantaleon & 658 & 657 & 666 & 775 & 794 & 815 & 855 & 30 \\
\hline
\end{tabular}

* Muttenz 18502222 E., abzüglich 9oo E. von Birsfelden, das $187+$ von Muttenz getrennt wurde.

6 Berufstätige 1950

\begin{tabular}{|c|c|c|c|c|c|c|c|c|c|}
\hline ufstätige 1950 & $\mathrm{~L}^{*}$ & $\%$ & $\mathrm{I}+\mathrm{H}^{*}$ & $0 \%$ & $\mathrm{G}, \mathrm{V}^{*}$ & $\%$ & $\mathrm{Ue}^{*}$ & $\%$ & $\begin{array}{l}\text { Total } \\
=100^{\circ} \%\end{array}$ \\
\hline Arlesheim & 56 & 3 & 845 & 46 & 415 & 23 & $50 t$ & 28 & 1820 \\
\hline Dornach & 48 & 3 & 965 & 55 & 313 & 18 & 416 & 24 & $17+2$ \\
\hline Reinach & 79 & 5 & 885 & 56 & 384 & 24 & 227 & 15 & 1575 \\
\hline Grellingen & 24 & 4 & 383 & 69 & 98 & 18 & 49 & 9 & 554 \\
\hline Muttenz & 98 & 3 & 1609 & 50 & 1037 & 32 & 467 & 15 & 3211 \\
\hline Pratteln & 105 & 3 & 1961 & 63 & 685 & 22 & 376 & 12 & 3127 \\
\hline Gempen & 66 & 42 & 65 & 42 & 15 & 10 & 1o & 6 & 156 \\
\hline Hochwald & 97 & 48 & 76 & 38 & 15 & 7 & 15 & 7 & 203 \\
\hline Seewen & 135 & 35 & 188 & 50 & 24 & 6 & 34 & 9 & 381 \\
\hline Büren & 96 & 32 & 167 & 56 & 23 & 8 & 11 & 4 & 297 \\
\hline Nuglar-St. Pantaleon & 71 & 18 & 245 & 64 & 47 & 12 & 23 & 6 & 386 \\
\hline
\end{tabular}

* $\mathrm{L}=$ Landwirtschaft $\mathrm{I}+\mathrm{H}=$ Industrie und Handwerk $\mathrm{G}, \mathrm{V}=$ Gastgewerbe, Handel und Verkehr $\mathrm{Ue}=$ andere Berufe.

7 Pendler ausgewählter Gemeinden 1950

\begin{tabular}{|c|c|c|c|c|c|c|c|}
\hline \multirow{4}{*}{$\begin{array}{l}\text { Arlesheim } \\
\text { Dornach }\end{array}$} & \multirow{4}{*}{$\begin{array}{l}\quad 1 \\
\text { Berufstätige } \\
1820 \\
1742\end{array}$} & \multirow{2}{*}{\multicolumn{2}{|c|}{$\begin{array}{c}2 \\
\text { Einpendler } \\
0 \% \text { von } 1\end{array}$}} & \multirow{2}{*}{\multicolumn{2}{|c|}{$\begin{array}{r}4 \quad 5 \\
\text { Auspendler } \\
\% \text { von } 1\end{array}$}} & \\
\hline & & & & & & $\begin{array}{c}\text { Ausp } \\
\text { Basel }\end{array}$ & $\begin{array}{l}\text { endler nach } \\
\% \text { von } 4\end{array}$ \\
\hline & & 559 & 30,7 & 735 & 40,4 & 484 & 65,8 \\
\hline & & 668 & 38,3 & 559 & 32,0 & 267 & 47,8 \\
\hline Muttenz & 3211 & 1279 & 39,8 & 1471 & 45,8 & 1082 & 73,5 \\
\hline Pratteln & 3127 & 2031 & 64,9 & 769 & 24,6 & 376 & 49,0 \\
\hline Gempen & 156 & o & 0,0 & 63 & 40,4 & 15 & 23,8 \\
\hline Hochwald & 203 & o & 0,0 & 55 & 27,1 & 13 & 23,6 \\
\hline
\end{tabular}

\title{
ÜBER DILUVIALE ABLAGERUNGEN AUS DER UMGEBUNG VON CHIASSO
}

\section{HANS RENFER}

Der vorliegende Bericht stellt die Zusammenfassung des glaziologischen Teiles einer im Entstehen begriffenen Arbeit über die V.di Muggio dar. Auf Grund zahlreicher neuer und z.T. tiefreichender Aufschlüsse konnte nachgewiesen werden, daß die Diluvialablagerungen im Mendrisiotto älter sind, als bisher allgemein angenommen wurde, und daß die jüngste Eiszeit nur noch durch vereinzelte Moränen mit Sicherheit nachgewiesen werden kann.

Der als mindeleiszeitlich zu datierende Ferretto stellt im Varesotto und Mendrisiotto die älteste nachweisbare Quartärablagerung dar. Nach NANgERoni (1954) ist E Bizzòzero (3 km SE Varese) am Abhang gegen die Olona auf oligo-/miozäner Molasse 
eine Überlagerung von Günz, Günz/Mindel-Schottern, Mindel und Riß vorhanden. Nach unserer Auffassung liegt aber nicht durchgehend stratigraphische Überlagerung, sondern teilweise Anlagerung vor. Ein von Rißresten bedeckter Mindelsockel liegt direkt auf der Molasse. Der Mindelmoräne liegen Schotter, diesen Würmgrundmoräne an. Letztere wird von NANGeroni als Günz beurteilt, doch schreibt er selbst: «Si potrebbe cioè pensare che sia morenico recente (tanto appare fresco!) ...»(1954, S. 21).

Während der Mindelzeit bildete sich durch die Ablagerungen der Capolago- und Comoarme des Addagletschers eine mächtige Moränebarriere, die zwischen Tradate und Appiano-Gentile zutage tritt, in unserem Untersuchungsgebiet im Straßeneinschnitt $\mathrm{N}$ Binago und als isoliertes nördlichstes Vorkommen an der Straße Viggiù - Clivio (Waldrand WNW Pt. 460 ; nach LK 1373) zu sehen ist. Weit auf diese Mindelablagerungen stießen die Rißgletscher vor. Als sie so weit abgeschmolzen waren, daß sich die einzelnen Zungen abzuzeichnen begannen, eventuell auch während eines neuen Eisvorstoßes, entstanden zwischen Stabio und Gaggiolo durch den Capolagogletscher zwei oder drei und im Faloppio zwischen Casanova - Olgiate Comasco und Uggiate-Parè durch einen Ausläufer des Comogletschers drei Stirnmoränen. E der Faloppio-Rißmoränen ist eine gegen NE geneigte Schotterebene festzustellen, welche die Ortschaften Camnago, Gaggino und Trévano trägt. Diese Schotter sind von etwas Rißmaterial, in der Kiesgrube $400 \mathrm{~m}$ E Trévano zudem auch von Würmgrundmoräne bedeckt und schließen wahrscheinlich an die drei Faloppiomoränen an, könnten aber auch unter diesen durchziehen. Diese Schotter sind mit der Rißvereisung in Verbindung zu bringen, die Deltaschotter der Breggia bei Balerna eventuell zeitlich mit ihnen zu parallelisieren. Wohl zum gleichen Gletscherstand wie die erwähnten Faloppio- und Stabiomoränen gehört die Rißmoräne, welche von der Landesgrenze bei Sagno über Morbio Superiore nach Somazzo verfolgt werden kann. Weitere Rißrückzugsstadien hinterließen die Moränen an der Breggia W Morbio Inferiore und die Wallmoräne von Vacallo - S. Simone, die in ihrem südlichsten Teil von Grundmoränen der letzten Eiszeit bedeckt ist. Die von Würmmaterial überzogene Rißmoräne von Corteglia (S Mendrisio) gehört zusammen mit der Seitenmoräne vom Museo Vela (N Ligornetto) bis N Rancate zur innersten Stirnmoräne von Stabio. Die Spuren der Würmvergletscherung sind spärlich, doch kann nachgewiesen werden, daß während des Würmmaximums die Eismassen entweder bis an die eben beschriebenen Rückzugsmoränen vorstießen (Morbio Superiore, Faloppio) oder sogar über diese hinaus reichten (Würmmoräne von Clivio, NNW Stabio).

Capolago- und Comogletscher erreichten während des heute nachweisbaren Maximalstandes am Alpenrand die Höhe von 800 bis $900 \mathrm{~m}$ ü. M., bei Mendrisio eine Mächtigkeit von $440 \mathrm{~m}$ und im Becken von Chiasso eine solche von $470 \mathrm{~m}$. Die Eismächtigkeit bei Morbio Superiore betrug $310 \mathrm{~m}$, und eine Eiszunge stie $\beta$ von hier aus, bei einem durchschnittlichen Gefälle des Talbodens von 46\% und einer im entgegengesetzten Sinne geneigten Eisoberfläche, deren Gefälle lediglich $7 \%$ betrug, $4,3 \mathrm{~km}$ talaufwärts, bis Muggio im Haupttal und bis Mobia im V. della Crotta. Gleichzeitig erfolgte aus dem Intelvi ein Eisvorstoß bis ins Quellgebiet der Breggia bei A. d'Orimento und zwischen S. Gordona und P. della Croce ein solcher in die Vallaccia.

\section{ALCUNi DEPOSITI DILUVIALI NEI DINTORNI DI CHIASSO}

Nel corso di un lavoro morfologico sulla V. di Muggio sono stati riesaminati i depositi glaciologici nei dintorni di Chiasso. Nella regione del rio Faloppia, nel bacino di Chiasso e presso Stabio prevale il morenico rissiano, spesso sotto forma di morenico a cordoni, mentre più rari sono i resti morenici dell'ultima glaciazione. Nel bacino di Chiasso il ghiaccio raggiunse uno spessore massimo di $490 \mathrm{~m}$, e di questa massa una lingua arrivò fino a Muggio, altre trasfluirono dall'Intelvi nell'alta valle della Breggia presso Orimento e nella Vallaccia tra S. Gordona e P. della Croce. 


\title{
LITERATUR
}

Annaheim H. 1936. Die Landschaftsformen des Luganerseegebietes. Geogr. Abhandlungen. Dritte Reihe Heft 8, Stuttgart. Saibene C. 1950. L'anfiteatro morenico del rio Faloppia (Como). Bollettino del comitato glaciologico italiano. N. I = II serie, Torino. Nangeroni G. 195t. I ter*reni pleistocenici dell'anfiteatro morenico del Verbano e del territorio varesino. Atti della Soc. It. di Sc. Nat. e del museio civico di storia nat. in Milano. Vol. 93, fasc. I-II. Milano.

\section{VERÄNDERUNGEN DES GOLFES VON THESSALONIKI}

\author{
LeONidAs Eumorphopulos
}

Wie die meisten Golfe der Erde unterlag auch derjenige von Thessaloniki in Nordgriechenland im Lauf der Zeit wesentlichen Veränderungen durch Verlandungsvorgänge. Im folgenden sei versucht, auf Grund verschiedener Untersuchungen eine Vorstellung davon zu bieten, wie diese Vorgänge sich vollzogen und welche Folgen sie für das Relief des Golfes gehabt haben.

\section{GESCHICHTLICHES}

Die historisch-geographischen Karten vom Golf von Thessaloniki zeigen, daß um 500 v. Chr. das Meer sich weit nach Westen bis an den Fuß der Gebirge erstreckte, so daß Pella, die Hauptstadt Philipps von Makedonien und Alexanders des Großen, eine Küstenstadt war. Die Stadt Skydra lag ca. $5 \mathrm{~km}$, Verria ca. $10 \mathrm{~km}$ vom Meer entfernt. Die Städte Termae und Sindos an der Ostküste dagegen waren Häfen. Die Werke der alten Schriftsteller bestätigen dies und erwähnen, daß gegenüber der Küstenstadt Pella sich die Insel Phakos befunden habe, in deren Festung die makedonischen Könige ihre Schätze aufbewahrten. Die Ruinen von Pella wurden 1959 entdeckt und sind in Ausgrabung begriffen.

Der Geologe STAnley Casson hat die Fortschritte der Zuschüttung der Westbucht des Golfes von Thessaloniki kartographisch festgehalten, wo vor allem der Fluß Axios wirkte, während an der Südküste der Haliakmon sedimentierte. Zwischen beiden Flüssen wurde Geschiebe vieler Wildbäche von den Bergen Païkos und Wermion abgelagert, wodurch um 100 v. Chr. der Westteil des Golfes von Thessaloniki abgeschnitten wurde, weil die Deltas von Axios und Haliakmon verschmolzen. Es bildete sich der Binnensee von Ludias. Da der Geschiebetransport fortdauerte, verkleinerte dieser sich und verwandelte sich 1925 in den schilfbedeckten und von Sümpfen umgebenen Süßwassersee von Jannitsa. Sein Wasserstand betrug $4,5-5,0 \mathrm{~m}$ über dem mittleren Meeresniveau, die größte Tiefe 5,o m. Die westliche Bucht war also bereits zu dieser Zeit stark zugeschüttet. Nunmehr, nach weiterer Verlandung und Setzung des Bodens um $1,5-2 \mathrm{~m} \mathrm{am}$ niedrigsten Punkt in Seemitte, ist der Boden um $1,5 \mathrm{~m}$ höher als das mittlere Meeresniveau. Das Wasser des Sees floß durch den Ludias-Fluß zum Meer. An der Ostküste fehlen größere Flüsse, und der Anthemuntos-Fluß führt nicht so viel Schwebstoffe mit, daß sie wesentlich verändert würde.

Naturgemäß hätte die andauernde Verlandung des Jannitsa-Sees dessen völliges Verschwinden zur Folge gehabt; doch wurde sie durch künstliche Eingriffe erheblich modifiziert. Im Auftrage des griechischen Staates ging die Gesellschaft «Foundation»daran, durch eine größere Reihe hydraulischer Werke die Küstenebene vor den Fluß- und Wildbachüberschwemmungen zu schützen und durch Austrocknung des Jannitza- und anderer Seen und Sümpfe Agrarland zu gewinnen. Eine der wichtigsten Arbeiten war die Schaffung eines $18 \mathrm{~km}$ langen neuen Bettes des Axios von der Brücke der Eisenbahn Thessaloniki-Athen bis zum Meer. Um künftige Überschwemmungen zu vermeiden, errichtete man parallel dazu in einem Abstand von 1 too m Dämme. Damit wurde die Stadt Thessaloniki selbst vor einer Wirtschaftskatastrophe gerettet, da sie sonst innerhalb von etwa 5o Jahren durch die Ablagerungen des Axios vom Meer getrennt worden wäre. Ein zweites großes hydraulisches Werk war die Regulierung des Haliakmon, die ebenfalls mit der Konstruktion eines (linksseitigen) Dammes verbunden war. Weiter bedeutete der Bau des Sammelkanals Wermion am Fuß des gleichnamigen Gebirges einen wirksamen Schutz der Ebene vor den Wildbächen desselben, die nun nach dem Haliakmon abgeleitet werden. Auch an der Nordseite des Jannitza-Sees wurden zahlreiche Wildbäche reguliert, deren Wasser der Ludias-Fluß und der Wardarobaschi-Kanal aufnehmen. Von den Entwässerungen ist die Trockenlegung des JannitzaSees und der benachbarten Sümpfe die wichtigste. Im ganzen veränderte sich mit diesen Werken die Situation der Thessaloniki-Ebene aufs gründlichste. Außerdem wurden neue Bedingungen für 